\title{
Metainferential Reasoning on Strong Kleene Models
}

\author{
Andreas Fjellstad ${ }^{1}$
}

Received: 15 June 2020 / Accepted: 19 June 2021 / Published online: 9 August 2021

(C) The Author(s) 2021

\begin{abstract}
Barrio et al. (Journal of Philosophical Logic, 49(1), 93-120, 2020) and Pailos (Review of Symbolic Logic, 13(2), 249-268, 2020) develop an approach to define various metainferential hierarchies on strong Kleene models by transferring the idea of distinct standards for premises and conclusions from inferences to metainferences. In particular, they focus on a hierarchy named the $\mathbb{S T}$-hierarchy where the inferential logic at the bottom of the hierarchy is the non-transitive logic ST but where each subsequent metainferential logic 'says' about the former logic that it is transitive. While Barrio et al. (2020) suggests that this hierarchy is such that each subsequent level 'in some intuitive sense, more classical than' the previous level, Pailos (2020) proposes an extension of the hierarchy through which a 'fully classical' metainferential logic can be defined. Both Barrio et al. (2020) and Pailos (2020) explore the hierarchy in terms of semantic definitions and every proof proceeds by a rather cumbersome reasoning about those semantic definitions. The aim of this paper is to present and illustrate the virtues of a proof-theoretic tool for reasoning about the $\mathbb{S T}$-hierarchy and the other metainferential hierarchies definable on strong Kleene models. Using the tool, this paper argues that each level in the $\mathbb{S T}$-hierarchy is non-classical to an equal extent and that the 'fully classical' metainferential logic is actually just the original non-transitive logic ST 'in disguise'. The paper concludes with some remarks about how the various results about the $\mathbb{S T}$-hierarchy could be seen as a guide to help us imagine what a non-transitive metalogic for ST would tell us about ST. In particular, it teaches us that ST is from the perspective of ST as metatheory not only non-transitive but also transitive.
\end{abstract}

Keywords Non-transitive logic $\cdot$ Metainferential hierarchies $\cdot$ Labelled sequent calculus $\cdot$ Nested sequent calculus $\cdot$ Strict-tolerant metatheory

The research for this paper was supported by the Research Council of Norway under grant number 262837/F10 (co-funded by the European Union's Seventh Framework Programme for research, technological development and demonstration under Marie Curie grant agreement no 608695).

Andreas Fjellstad

afjellstad@gmail.com

1 Department of Philosophy, University of Bergen, Bergen, Norway 


\section{Introduction}

[1] and [2] develop an approach to define various metainferential hierarchies on strong Kleene models by transferring the idea of mixed inferences to the metainferential level. In particular, their investigations reveal that a particular hierarchy named ST is definable where the inferential logic at the bottom of the hierarchy is the non-transitive logic ST advocated by [3] and [4] but where each subsequent metainferential logic 'says' about the former logic that it is transitive. While [1] suggest that this hierarchy provides metainferential logics where each subsequent level is 'in some intuitive sense, more classical than' the previous level, [2] proposes an extension of the hierarchy through which a 'fully classical' metainferential logic can be defined. Both [1] and [2] explore the hierarchies from a semantic perspective and every proof proceeds by a rather cumbersome reasoning about those semantic definitions.

The primary aim of this paper is to develop and illustrate the use of a prooftheoretic tool obtained by combining ideas from nested sequent calculi with labelled sequent calculi for reasoning about $\mathrm{ST}$ and the other metainferential hierarchies definable on strong Kleene models. To that purpose, Section 2 presents the approach to metainferential hierarchies on strong Kleene models developed by [1] and Section 3 presents a "labelled nested" sequent calculus based on the definitions provided in Section 2. This tool is then employed to make some remarks about [1]'s metainferential hierarchy ST and [2]'s 'fully classical' metainferential logic. In particular, it is shown in Section 4 that each level in the $\mathbb{S T}$ hierarchy is non-classical to an equal extent, a result which is extended in Section 5 to the 'fully classical' metainferential logic presented by [2]. Moreover, it is also shown that every metainference of the 'fully classical' metainferential logic is equivalent to an inference of the original non-transitive logic ST, and that the former is thus the latter 'in disguise'. Finally, the paper proposes in Section 6 that the hierarchy $\mathbb{S T}$ can fruitfully be understood as a tool to help us imagine what ST would tell us about ST if ST is used as metatheory where the most interesting observation being that ST is from the perspective of ST both transitive and non-transitive.

\section{Language and Models}

This section presents the language and models that will form the basis for the proof theory.

Definition 2.1 (The language) Let $\mathcal{L}$ be a propositional language based on a countable set of propositional variables, a nullary connective $\lambda$, a unary connective $\neg$ and the binary connective $\vee$. Let FORML $L_{\mathcal{L}}$ be the set of formulas of $\mathcal{L}$.

We shall use upper case Latin letters $A, B$ etc as metalinguistic variables for formulas in general and lower case Latin letters $p, q$ etc as metalinguistic variables for propositional variables. 
In addition to having formulas that are assigned values and can satisfy certain standards on strong Kleene models, we are interested in metainferences as objects that can satisfy certain appropriate standards on strong Kleene models, that is, as objects that can feature in a satisfaction relation. Following [1] we will define a hierarchy of metainferential objects as follows using the notation $[\ldots \Rightarrow \ldots]$ :

Definition 2.2 (The metainferential objects)

- If $A_{1}, \ldots, A_{n}$ and $B_{1}, \ldots, B_{m}$ are formulas of $\mathcal{L}$, then $\left[A_{1}, \ldots, A_{n} \Rightarrow\right.$ $\left.B_{1}, \ldots, B_{m}\right]$ is a metainferential object of level 0

- If $X_{1}, \ldots, X_{n}$ and $Y_{1}, \ldots, Y_{m}$ are metainferential objects of level $k$ then $\left[X_{1}, \ldots, X_{n} \Rightarrow Y_{1}, \ldots, Y_{m}\right]$ is a metainferential object of level $k+1$.

Metainferential objects can thus be seen as binary connectives that applies to sets of objects. They are however not part of $\mathcal{L}$ even if they contain objects from $\operatorname{FORM}_{\mathcal{L}}$. Moreover, while one might be tempted to add numerals to the objects in order to identify its level, this is not necessary since $X_{i}$ in $\left[X_{1}, \ldots, X_{n} \Rightarrow Y_{1}, \ldots, Y_{m}\right]$ will be an object of the previous level if the level is $>0$ or a formula if the level is 0 .

Definition 2.3 (Strong Kleene valuations) A function $\mathcal{V}:$ FORML $_{\mathcal{L}} \rightarrow\left\{1, \frac{1}{2}, 0\right\}$ is a strong Kleene valuation just in case $\mathcal{V}(\lambda)=\frac{1}{2}$ and the following conditions are satisfied for every complex formula:

$$
\mathcal{V}(A \vee B)=\left\{\begin{array}{ll}
1 & \mathcal{V}(A)=1 \text { or } \mathcal{V}(B)=1 \\
0 & \mathcal{V}(A)=0 \text { and } \mathcal{V}(B)=0 \\
\frac{1}{2} & \text { otherwise }
\end{array} \quad \mathcal{V}(\neg A)= \begin{cases}1 & \mathcal{V}(A)=0 \\
0 & \mathcal{V}(A)=1 \\
\frac{1}{2} & \mathcal{V}(A)=\frac{1}{2}\end{cases}\right.
$$

Following [5], a formula on trivalent models can be either strictly or tolerantly satisfied. This is made precise as follows:

Definition 2.4 (Satisfaction of formulas)

$-\quad \mathcal{V} \Vdash_{s} A$ if and only if $\mathcal{V}(A)=1$

$-\mathcal{V} \Vdash_{t} A$ if and only if $\mathcal{V}(A) \in\left\{1, \frac{1}{2}\right\}$

[1] extends the notion of satisfaction from formulas to metainferential objects using a hierarchy of metainferential standards based on the strict-tolerant distinction. Informally presented, the idea is as follows:

- A formula can satisfy one out of two standards, $s$ and $t$.

- A metainference of level 0 can satisfy one or more out of four standards, $s t, t s$, $s s$ and $t t$.

- A metainference of level 1 can satisfy one or more out of sixteen standards:

$$
\begin{array}{ll}
- & \text { stst, tsst, ssst, ttst } \\
- & \text { stts, tsts, ssts, ttts } \\
- & \text { stss,tsss, sss, ttss }
\end{array}
$$


- $\quad$ stt, tstt, sstt, ttt

- A metainference of level 2 can satisfy one or more out of 256 standards which we shall not list.

An inductive definition can thus be given as follows:

Definition 2.5 (The standards)

- $\quad s$ and $t$ are formula standards.

- if $x$ and $y$ are formula-standards, then $x y$ is a metainferential standard of level 0 .

- if $x$ and $y$ are metainferential standards of level $n$, then $x y$ is a metainferential standard of level $n+1$.

Following [1], the notion of satisfaction can now be extended as follows:

Definition 2.6 (Satisfaction of metainferences) If $[\Gamma \Rightarrow \Delta]$ is a metainferential object of level $n$ and $x y$ a metainferential standard of level $n$, then,

$$
\mathcal{V} \Vdash_{x y}[\Gamma \Rightarrow \Delta] \text { iff some } X \in \Gamma, \mathcal{V} \nVdash_{x} X \text { or some } Y \in \Delta, \mathcal{V} \Vdash_{y} Y
$$

Finally, validity for the various inferential and metainferential logics is now defined as follows:

Definition 2.7 (Validity) $\Gamma \vDash_{x y} \Delta$ iff for every $\mathcal{V}, \mathcal{V} \Vdash_{x y}[\Gamma \Rightarrow \Delta]$

Unsurprisingly, the various inferential and metainferential logics definable on strong Kleene models recently discussed in the literature fall out of this definition. We shall in general refer to a particular logic through its standard, e.g. the logic st or the logic $t s s t$. There is however one logic definable on strong Kleene models that is not captured by this approach and which on occasion is discussed in the literature, e.g. by [6], namely that definable using $\leq$ as follows: $\Gamma \vDash \Delta$ iff every $\mathcal{V}$ is such that $\min (\mathcal{V}(A) \in \Gamma) \leq \max (\mathcal{V}(B) \in \Delta)$. This is an acceptable limitation considering the aim of this paper.

\section{The HST Calculus}

This section presents a sequent calculus representing metainferential hierarchies on strong Kleene valuations based on the definitions provided in the previous section. The hierarchical strict-tolerant calculus will be a labelled sequent calculus in the sense that the rules will not manipulate formulas directly as in the case of a standard sequent calculus, but rather labelled formulas and labelled metainferential objects. To that purpose we shall introduce one label for each standard to thereby obtain labelled formulas (e.g. $s: A$ ) and labelled metainferential objects of the form $x:[\Gamma \Rightarrow$ $\Delta$ ] where $\Gamma$ and $\Delta$ will be formulas if it is a metainferential object of level 0 and metainferential object of level $n$ if it is a metainferential object of level $n+1$. Since the calculus will thus contain expressions that look like standard sequents nested 
within each others, it can also rightly be described as a sequent calculus for nested sequents. It is thus a labelled nested sequent calculus.

While the calculus is straightforwardly modified to also include the addition of socalled "antivalidities" as introduced into the debate by [7] and thus also capture the arguments presented by [7], such modifications are purposely left out to keep things simpler and more straightforward. The reader with an interest in such issues is invited to make the appropriate amendments.

Definition 3.1 (Typed sequent expression)

- If $A$ is a formula and $x \in\{s, t\}$, then $x: A$ is a sequent expression of type $(0, x)$

- If $x: X_{1}, \ldots, x: X_{n}$ are sequent expressions of type $(\alpha, x)$ and $y: Y_{1}, \ldots, y: Y_{m}$ are of sequent expressions of type $(\alpha, y)$ then $x y:\left[X_{1}, \ldots, X_{n} \Rightarrow Y_{1}, \ldots, Y_{m}\right]$ is a sequent expression of type $(\alpha+1, x y)$.

For a sequent expression of type $(\alpha, x)$, we refer to $\alpha$ as the level and $x$ as the standard. We shall use $x: X$ to refer to an arbitrary sequent expression of any type. Moreover, we let $\mathbb{X}_{(\alpha, x)}$ and $\mathbb{Y}_{(\alpha, y)}$ designate finite multisets of sequent expressions of type $(\alpha, x)$ and $(\alpha, y)$ respectively. We also let $\mathbb{X}$ designate the multiset obtained by removing labels from the members of $\mathbb{X}_{(\alpha, x)}$, i.e. $\mathbb{X}=\left\{X \mid x: X \in \mathbb{X}_{(\alpha, x)}\right\}$. Note also that it follows from the notation that a metainference of level $n$ is represented by a sequent expression of level $n+1$.

The following are examples of typed sequent expressions.

$$
\begin{aligned}
& s t:[A, \neg A \vee B \Rightarrow B] \quad t t:[A \vee B \Rightarrow A, B] \\
& \text { tsst:[[A } \Rightarrow A],[\Rightarrow \neg A \vee B] \Rightarrow[A \Rightarrow B]] \\
& \text { ttst: }[\Rightarrow[\Rightarrow A],[A \Rightarrow]] \\
& \text { tsstttss: }[[[A \Rightarrow A],[\Rightarrow \neg A \vee B] \Rightarrow[A \Rightarrow B]] \Rightarrow[\Rightarrow[\Rightarrow A],[A \Rightarrow]]]
\end{aligned}
$$

As the examples suggest, it will be increasingly difficult to read the sequent expressions in order to decipher their level and thus their meaning. Luckily the construction is compositional and we are in general only interested in the inductive steps from level $n$ to level $n+1$.

Definition 3.2 (The HST calculus) Let HST be the sequent calculus obtained with the following rules where sequents are pairs of multisets of typed sequent expressions.

Initial sequents:

$$
s: p, \Gamma \Rightarrow \Delta, s: p \quad s: p, \Gamma \Rightarrow \Delta, t: p \quad t: p, \Gamma \Rightarrow \Delta, t: p
$$

Rules for sequent expressions of level 0 :

$$
\begin{aligned}
& \frac{x: A, \Gamma \Rightarrow \Delta \quad x: A, \Gamma \Rightarrow \Delta}{x: A \vee B, \Gamma \Rightarrow \Delta} \vee \mathrm{L} \quad \frac{\Gamma \Rightarrow \Delta, x: A, x: B}{\Gamma \Rightarrow \Delta, x: A \vee B} \vee \mathrm{R} \\
& \frac{\Gamma \Rightarrow \Delta, x: A}{y: \neg A, \Gamma \Rightarrow \Delta} \neg \mathrm{L}(x \neq y) \quad \frac{x: A, \Gamma \Rightarrow \Delta}{\Gamma \Rightarrow \Delta, y: \neg A} \neg \mathrm{R}(x \neq y) \\
& \overline{s: \lambda, \Gamma \Rightarrow \Delta}^{\lambda \mathrm{L}} \quad \overline{\Gamma \Rightarrow \Delta, t: \lambda}^{\lambda \mathrm{R}}
\end{aligned}
$$


Rules for sequent expressions of level $>0$ :

$$
\begin{gathered}
\Gamma \Rightarrow \Delta, x: X \quad \text { for every } x: X \in \mathbb{X}_{(\alpha, x)} \quad y: Y, \Gamma \Rightarrow \Delta \quad \text { for every } y: Y \in \mathbb{Y}_{(\alpha, y)} \\
x y:[\mathbb{X} \Rightarrow \mathbb{Y}], \Gamma \Rightarrow \Delta \\
\frac{\mathbb{X}_{(\alpha, x)}, \Gamma \Rightarrow \Delta, \mathbb{Y}_{(\alpha, y)}}{\Gamma \Rightarrow \Delta, x y:[\mathbb{X} \Rightarrow \mathbb{Y}]}[\Rightarrow] \mathrm{R}
\end{gathered}
$$

This calculus is well-behaved from the perspective of structural proof theory as elucidated by [8] and [9]. In particular, we have the following lemmas and theorems where each proof is obtainable through a straight-forward adaptation of the corresponding proof in [9].

Definition 3.3 (Derivation height) The height of a HST-derivation $\mathcal{D}, \mathcal{H}(\mathcal{D})$ is defined inductively as follows.

- If $\mathcal{D}$ is an initial sequent or conclusion of a zero-premise rule, then $\mathcal{H}(\mathcal{D})=0$.

- If $\mathcal{D}$ is obtained with an $\alpha$-premise rule from derivations $\mathcal{D}_{i}$ for $0 \leq i<\alpha$, then $\mathcal{H}(\mathcal{D})=\sup _{i<\alpha}\left(\mathcal{H}\left(\mathcal{D}_{i}\right)+1\right)$

We'll say that a rule is height-preservingly admissible (HP-admissible) just in case whenever there is a derivation of the premise-sequent with height $n$ then there is a derivation of the conclusion-sequent with height $\leq n$.

Lemma 3.4 (Weakening and contraction) The following rules are HP-admissible in HST:

$$
\frac{\Gamma \Rightarrow \Delta}{\Gamma^{\prime}, \Gamma \Rightarrow \Delta, \Delta^{\prime}} \quad \frac{x: X, x: X, \Gamma \Rightarrow \Delta}{x: X, \Gamma \Rightarrow \Delta} \quad \frac{\Gamma \Rightarrow \Delta, x: X, x: X}{\Gamma \Rightarrow \Delta, x: X}
$$

Proof The proofs are obtained by slight modifications on the proofs of proposition 4.4 and theorem 4.12 in [9].

Lemma 3.5 (Inversion) The inversion of each primitive HST-rule is HP-admissible in HST.

Proof Proof is a modification of proposition 4.11 in [9].

Definition 3.6 (Formula complexity) The complexity of a $\mathcal{L}$-formula $A,|\mathrm{~A}|$, is defined inductively as follows:

- If $A$ is an atomic formula, then $|A|=0$

- If $A$ is of the form $\neg B$, then $|A|=|B|+1$

- If $A$ is of the form $B \vee C$, then $|A|=|B|+|C|+1$.

Definition 3.7 (Expression weight) Suppose that $x: X$ is a sequent expression. Then the weight of $X, \mathcal{W}(X)$, is defined as follows: if $X$ is a formula $A$, then $\mathcal{W}(A)=|\mathrm{A}|$ and if $X$ is a metainferential object $[\Gamma \Rightarrow \Delta]$, then $\mathcal{W}([\Gamma \Rightarrow \Delta])=$ $\sum_{Y \in \Gamma}(\mathcal{W}(Y))+\sum_{Y \in \Delta}(\mathcal{W}(Y))+1$. 
Theorem 3.8 (Cut) The following rule is admissible in HST:

$$
\frac{\Gamma \Rightarrow \Delta, x: X \quad x: X, \Gamma^{\prime} \Rightarrow \Delta^{\prime}}{\Gamma, \Gamma^{\prime} \Rightarrow \Delta, \Delta^{\prime}}
$$

Proof By double induction on the weight of $X$ and the sum of the heights of the derivations of the premise-sequents. See theorem 4.13 in [9].

Definition 3.9 (Sequent validity) A sequent $\Gamma \Rightarrow \Delta$ is valid just in case there is no valuation $\mathcal{V}$ such that

- for every typed sequent expression $x: X \in \Gamma, \mathcal{V} \Vdash_{x} X$

- for every typed sequent expression $y: Y \in \Delta, \mathcal{V} \nVdash_{y} Y$

Theorem 3.10 (Completeness) A sequent $\Gamma \Rightarrow \Delta$ is valid if and only if $\Gamma \Rightarrow \Delta$ is derivable in HST.

Proof The right-to-left direction proceeds as usual by induction on the height of a derivation. The left-to-right direction proceeds as usual via the construction of a reduction tree for every underivable sequent from which a countermodel for that sequent is extracted. We present here a few details from the latter proof.

Assume that $\Gamma \Rightarrow \Delta$ is underivable. It follows that we can construct a tree above it by applying the rules of the HST calculus backwards until each branch ends with a sequent containing only labelled propositional variables and labelled $\lambda$ 's. At least one branch will be such that the leaf is not an initial sequent or a zero-premise rule of the HST calculus. We pick such a branch $\mathcal{B}$ and define a function $\mathcal{V}$ from the set of propositional variables of $\mathcal{L}$ to $\left\{1,0, \frac{1}{2}\right\}$ as follows where $\Gamma^{\prime} \Rightarrow \Delta^{\prime}$ is the leaf-sequent of $\mathcal{B}$ :

- for every $s: p$ in $\Gamma^{\prime}, \mathcal{V}(p)=1$,

- for every $t: p$ in $\Delta^{\prime}, \mathcal{V}(p)=0$,

- otherwise, $\mathcal{V}(p)=\frac{1}{2}$.

The definition of $\mathcal{V}$ is extended to complex formulas and $\lambda$ in accordance with definition 2.3. The satisfaction relation $\Vdash$ is defined in accordance with definitions 2.4 and 2.6. We can now show by induction on the complexity of a formula that for every $\Gamma^{\prime \prime} \Rightarrow \Delta^{\prime \prime} \in \mathcal{B}$ :

- If $x: A \in \Gamma^{\prime \prime}$ then $\mathcal{V} \Vdash_{x} A$.

- If $x: A \in \Delta^{\prime \prime}$ then $\mathcal{V} \nVdash_{x} A$.

It is left to show by an induction on the set of standards from definition 2.5 that also the following statements hold for every $\Gamma^{\prime \prime} \Rightarrow \Delta^{\prime \prime} \in \mathcal{B}$ :

- If $x y:\left[\Gamma^{\prime} \Rightarrow \Delta^{\prime}\right] \in \Gamma^{\prime \prime}$ then $\mathcal{V} \Vdash_{x y}\left[\Gamma^{\prime} \Rightarrow \Delta^{\prime}\right]$.

- If $x y:\left[\Gamma^{\prime} \Rightarrow \Delta^{\prime}\right] \in \Delta^{\prime \prime}$ then $\mathcal{V} \nVdash_{x y}\left[\Gamma^{\prime} \Rightarrow \Delta^{\prime}\right]$. 
With $\Gamma \Rightarrow \Delta \in \mathcal{B}$, it follows that for every typed sequent expression $x: X \in \Gamma$, $\mathcal{V} \Vdash_{x} X$ and for every typed sequent expression $y: Y \in \Delta, \mathcal{V} \nVdash_{y} Y . \mathcal{V}$ is thus a countermodel for the sequent $\Gamma \Rightarrow \Delta$.

Corollary $3.11 \Rightarrow x y:[\Gamma \Rightarrow \Delta]$ is derivable if and only if $\Gamma \vDash_{x y} \Delta$

Before we dive into the perhaps more serious applications of this proof-theoretic tool, we shall first provide a few illustrations of its immediate usefulness. As a first curiosity we shall show that the inferential logic defined with the standard $s t$ is nontransitive using the admissibility of cut.

Lemma 3.12 (Nontriviality) For every label $x y$, sequent $\Rightarrow x y:[\Rightarrow]$ is not derivable.

Proof The empty sequent follows by inversion, but the empty sequent is excluded by design.

Lemma 3.13 (st is inconsistent) The sequents $\Rightarrow s t:[\Rightarrow \lambda]$ and $\Rightarrow s t:[\lambda \Rightarrow]$ are derivable.

Proposition 3.14 (st is nontransitive) The sequent st: $[\lambda]$, st: $[\lambda \Rightarrow] \Rightarrow s t:[\Rightarrow]$ is underivable.

Proof If that sequent is derivable then theorem 3.8 and lemma 3.13 together imply that $\Rightarrow s t:[\Rightarrow]$ is derivable but this is excluded by lemma 3.12 .

With inspiration from [10], we have thus shown the nontransitivity of a logic typically defined proof-theoretically by rejecting cut using the admissibility of cut.

In fact, we actually have a tool which can be used as a "metasequent" calculus for the four logics $s t, t s, t t$ and $s s$. In the case of $s t$, for example, the following sequents are derivable:

$$
\begin{gathered}
\Rightarrow s t:[A, \Gamma \Rightarrow \Delta, A] \\
s t:[\Gamma \Rightarrow \Delta, A] \Rightarrow s t:[\neg A, \Gamma \Rightarrow \Delta] \\
s t:[A, \Gamma \Rightarrow \Delta] \Rightarrow s t:[\Gamma \Rightarrow \Delta, \neg A] \\
s t:[A, \Gamma \Rightarrow \Delta], s t:[B, \Gamma \Rightarrow \Delta] \Rightarrow s t:[A \vee B, \Gamma \Rightarrow \Delta] \\
s t:[\Gamma \Rightarrow \Delta, A, B] \Rightarrow s t:[\Gamma \Rightarrow \Delta, A \vee B]
\end{gathered}
$$

One can now use cut to obtain every st-validity, where then each application of cut corresponds to one application of a rule in the standard two-sided sequent calculus for $s t$ such as that presented by [3].

Moreover, we can use the sequent calculus to illustrate the relationships between the logics $s t, t s, s s$ and $t t$ familiar from the literature. For example, we can show 
that a certain instance of transitivity holds in $s t$ just in case a corresponding instance reflexivity holds in $t s$ with the following derivations:

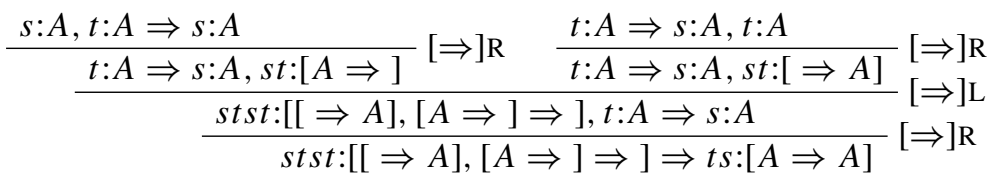

$$
\begin{aligned}
& \frac{t: A, s t:[A \Rightarrow] \Rightarrow t: A}{s t:[\Rightarrow A], s t:[A \Rightarrow] \Rightarrow t: A}[\Rightarrow] \mathrm{R} \quad \frac{s: A, s t:[\Rightarrow A] \Rightarrow t: A}{s: A, s t:[\Rightarrow A], s t:[A \Rightarrow] \Rightarrow}[\Rightarrow] \mathrm{R}
\end{aligned}
$$

To provide further familiar facts about the logics (and also for some propositions in the next section), the following lemma for transforming labels will be useful.

Lemma 3.15 (label transformation) (a) If A is a formula, then following rules are HP-admissible:

$$
\frac{t: A, \Gamma \Rightarrow \Delta}{s: A, \Gamma \Rightarrow \Delta} t / s L \quad \frac{\Gamma \Rightarrow \Delta, s: A}{\Gamma \Rightarrow \Delta, t: A} s / t R
$$

(b) If $X$ is a sequent expression of level 1, then the following rules are HPadmissible:

$$
\begin{array}{ll}
\frac{x t: X, \Gamma \Rightarrow \Delta}{x s: X, \Gamma \Rightarrow \Delta} x t / x s L & \frac{s x: X, \Gamma \Rightarrow \Delta}{t x: X, \Gamma \Rightarrow \Delta} s x / t x L \\
\frac{\Gamma \Rightarrow \Delta, t x: X}{\Gamma \Rightarrow \Delta, s x: X} t x / s x R & \frac{\Gamma \Rightarrow \Delta, x s: X}{\Gamma \Rightarrow \Delta, x t: X} x s / x t R
\end{array}
$$

Proof The proofs straightforwardly follow the general strategy in structural proof theory to show the height-preserving admissibility of a rule.

Regarding (a), we prove $\mathrm{t} / \mathrm{sL}$ and s/tR simultaneously. We here focus on $\mathrm{t} / \mathrm{sL}$.

Base case: Assume $0 \vdash t: A, \Gamma \Rightarrow \Delta$. If $A$ is a propositional variable and $t: A \in \Delta$, then $s: A, \Gamma \Rightarrow \Delta$ is also an initial sequent. If $\Gamma \Rightarrow \Delta$ is an initial sequent or an instance of zero-premise rule, then $s: A, \Gamma \Rightarrow \Delta$ is also an initial sequent or an instance of a zero-premise rule and thus $0 \vdash s: A, \Gamma \Rightarrow \Delta$ holds.

Inductive step: Assume $n+1 \vdash t: A, \Gamma \Rightarrow \Delta$. If $t: A$ is principal and of the form $\neg B$, it follows that $n \vdash \Gamma \Rightarrow \Delta, s: B$. By the inductive hypothesis we obtain $n \vdash$ $\Gamma \Rightarrow \Delta, t: B$ and by one application of $\neg L$ we obtain $n+1 \vdash t: \neg B, \Gamma \Rightarrow \Delta$. The case for $\vee \mathrm{L}$ is similar. If $t: A$ is not principal, the sequent is obtained with some $k$ premise rule $\mathcal{R}$ and it is thus the case that $n \vdash t: A, \Gamma^{i} \Rightarrow \Delta^{i}$ for every $i<k$. We apply the inductive hypothesis to obtain $n \vdash s: A, \Gamma^{i} \Rightarrow \Delta^{i}$ and one application of $\mathcal{R}$ delivers $n+1 \vdash s: A, \Gamma \Rightarrow \Delta$.

Regarding (b) we proceed as follows, focusing only on the case of tx/sxR. Assume $n+1 \vdash \Gamma^{\prime} \Rightarrow \Delta^{\prime}, t x:[\Gamma \Rightarrow \Delta]$. If $t x:[\Gamma \Rightarrow \Delta]$ is principal, then $n \vdash t: \Gamma, \Gamma^{\prime} \Rightarrow$ $\Delta^{\prime}, x: \Delta$. By (a) we obtain $n \vdash s: \Gamma, \Gamma^{\prime} \Rightarrow \Delta^{\prime}, x: \Delta$ and finally $n+1 \vdash \Gamma^{\prime} \Rightarrow$ $\Delta^{\prime}, s x:[\Gamma \Rightarrow \Delta]$ by the relevant rule for introducing the desired expression. If 
$t x:[\Gamma \Rightarrow \Delta]$ is not principal, then the sequent in question is obtained with some $k$ premise rule $\mathcal{R}$ and it is thus the case that $n \vdash \Gamma^{i} \Rightarrow \Delta^{i}, t x:[\Gamma \Rightarrow \Delta]$ for every $i<k$. We apply the inductive hypothesis to obtain $n \vdash \Gamma^{i} \Rightarrow \Delta^{i}, s x:[\Gamma \Rightarrow \Delta]$ and then apply $\mathcal{R}$ to obtain $n+1 \vdash \Gamma^{\prime} \Rightarrow \Delta^{\prime}, s x:[\Gamma \Rightarrow \Delta]$.

This lemma has two immediate corollaries. The first concerns the relationship between valid inferences in the four logics as familiar from [5].

Corollary 3.16 (Relationships between $s t, t s, s s$ and $t t$ ) The following sequents are derivable:

$$
t s: X \Rightarrow t t: X \quad t s: X \Rightarrow s s: X \quad t t: X \Rightarrow s t: X \quad s s: X \Rightarrow s t: X
$$

The second concerns the relationship between inferences of $s t$ and $t s$ on the one hand, and formulas that are tolerantly and strictly satisfied on the other hand.

Corollary 3.17 The following rules are admissible were $x \neq y$ :

$$
\begin{aligned}
& \frac{x y:\left[A_{1}, \ldots, A_{n} \Rightarrow_{1} B_{1}, \ldots, B_{m}\right], \Gamma \Rightarrow \Delta}{y: \neg A_{1} \vee \ldots \vee \neg A_{n} \vee B_{1} \vee \ldots \vee B_{m}, \Gamma \Rightarrow \Delta} \\
& \frac{\Gamma \Rightarrow \Delta, x y:\left[A_{1}, \ldots, A_{n} \Rightarrow_{1} B_{1}, \ldots, B_{m}\right]}{=\Gamma \Rightarrow \Delta, y: \neg A_{1} \vee \ldots \vee \neg A_{n} \vee B_{1} \vee \ldots \vee B_{m}}
\end{aligned}
$$

Proof By inversion and straightforward applications of the relevant rules.

An immediate consequence of that corollary is the following result by [11] and [12]:

Proposition 3.18 The following rule is admissible:

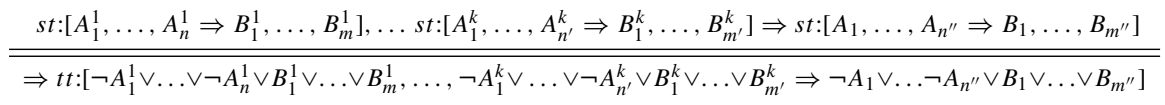

The corresponding result for $t s$ as presented by for example [13] is obviously also available:

Proposition 3.19 The following rule is admissible:

$$
\frac{t s:\left[A_{1}^{1}, \ldots, A_{n}^{1} \Rightarrow B_{1}^{1}, \ldots, B_{m}^{1}\right], \ldots t s:\left[A_{1}^{k}, \ldots, A_{n^{\prime}}^{k} \Rightarrow B_{1}^{k}, \ldots, B_{m^{\prime}}^{k}\right] \Rightarrow t s:\left[A_{1}, \ldots, A_{n^{\prime \prime}} \Rightarrow B_{1}, \ldots, B_{m^{\prime \prime}}\right]}{\overline{\Rightarrow s s:\left[\neg A_{1}^{1} \vee \ldots \vee \neg A_{n}^{1} \vee B_{1}^{1} \vee \ldots \vee B_{m}^{1}, \ldots, \neg A_{1}^{k} \vee \ldots \vee \neg A_{n^{\prime}}^{k} \vee B_{1}^{k} \vee \ldots \vee B_{m^{\prime}}^{k} \Rightarrow \neg A_{1} \vee \ldots \neg A_{n^{\prime \prime}} \vee B_{1} \vee \ldots \vee B_{m^{\prime \prime}}\right]}}
$$

What is interesting here is not the fact that these propositions hold about the four logics $s t, t s, t t$ and $s s$, but the ease with which we have obtained them using proof analysis. 


\section{Approximating Classicality with the ST-Hierarchy?}

The results in the previous section concerned only inferences and metainferences, not inferences of metainferences and so forth. Given the generality of our calculus, it should be clear that we can also use it to prove facts about "higher-order" metainferences. To illustrate that we shall have a look at what we can say about the ST-hierarchy of metainferential standards presented by [1]. The basic idea with the hierarchy is to "reproduce" the "st-phenomenon" at a metainferential level by defining a hierarchy of metainferential standards where the standard for being a premise in a sound inference is stricter than the standard for being a conclusion. For our purposes, we can replicate the hierarchy of standards with labels using the following definition:

Definition 4.1 (The $\mathbb{S T}$-hierarchy) The set of labels $\mathbb{S T}$ is defined inductively as follows:

$-\quad s t \in \mathbb{S T}$

- If $x y \in \mathbb{S T}$ then $y x x y \in \mathbb{S T}$

The standard for metainferences of level 1 is thus $t s s t$ as opposed to the standard stst, and for metainferences of level 2 we have the standard sttstsst as opposed to the standard tssttsst. We will use $\mathrm{ST}_{n}$ to refer to the $n$th level in $\mathbb{S T}$, so that $\mathrm{ST}_{0}$ is $s t, \mathrm{ST}_{1}$ is $t$ sst, and so on.

What is interesting about $\mathbb{S T}$ according to [1] is that "in some intuitive sense, TS/ST is classical to a greater degree than ST", and moreover that we obtain at each $\mathrm{ST}_{n+1}$ a "metainferential" logic which is supposedly more similar to classical logic than $\mathrm{ST}_{n}$ because stage $n$ is according to stage $n+1$ transitive. To illustrate this, consider the following observation about $t$ sst:

Proposition 4.2 ( $t$ sst concerns a transitive logic) The following sequent is derivable:

$$
\Rightarrow \operatorname{tsst}:\left[[\Gamma \Rightarrow \Delta, A],\left[A, \Gamma^{\prime} \Rightarrow \Delta^{\prime}\right] \Rightarrow\left[\Gamma, \Gamma^{\prime} \Rightarrow \Delta, \Delta^{\prime}\right]\right]
$$

Proof We have for every formula $A$ a derivation of the following form:

$$
\frac{\frac{\vdots}{t: \Gamma \Rightarrow t: \Gamma} \underset{\frac{t s:[\Gamma \Rightarrow \Delta, A], t: \Gamma \Rightarrow s: \Delta, t: A}{s: \Delta \Rightarrow s: \Delta}}{\frac{s}{s \Rightarrow A \Rightarrow t: A}}[\Rightarrow] \mathrm{L} \quad \frac{\vdots}{t: \Gamma^{\prime} \Rightarrow t: \Gamma^{\prime}} \frac{\vdots}{s: \Delta^{\prime} \Rightarrow s: \Delta^{\prime}}}{[\Rightarrow] \mathrm{L} \frac{t s:[\Gamma \Rightarrow \Delta, A], t s:\left[A, \Gamma^{\prime} \Rightarrow \Delta^{\prime}\right], t: \Gamma, t: \Gamma^{\prime} \Rightarrow s: \Delta, s: \Delta^{\prime}}{t s:[\Gamma \Rightarrow \Delta, A], t s:\left[A, \Gamma^{\prime} \Rightarrow \Delta^{\prime}\right] \Rightarrow t s:\left[\Gamma, \Gamma^{\prime} \Rightarrow \Delta, \Delta^{\prime}\right]}}
$$

We then proceed as follows using label transformation:

$$
\frac{\frac{t s:[\Gamma \Rightarrow \Delta, A], t s:\left[A, \Gamma^{\prime} \Rightarrow \Delta^{\prime}\right] \Rightarrow t s:\left[\Gamma, \Gamma^{\prime} \Rightarrow \Delta, \Delta^{\prime}\right]}{t s:[\Gamma \Rightarrow \Delta, A], t s:\left[A, \Gamma^{\prime} \Rightarrow \Delta^{\prime}\right] \Rightarrow s s:\left[\Gamma, \Gamma^{\prime} \Rightarrow \Delta, \Delta^{\prime}\right]}}{\frac{t x:[\Gamma \Rightarrow \Delta, A], t s:\left[A, \Gamma^{\prime} \Rightarrow \Delta^{\prime}\right] \Rightarrow s t:\left[\Gamma, \Gamma^{\prime} \Rightarrow \Delta, \Delta^{\prime}\right]}{\operatorname{ts}:\left[\Gamma s t:\left[[\Gamma \Rightarrow \Delta, A],\left[A, \Gamma^{\prime} \Rightarrow \Delta^{\prime}\right] \Rightarrow\left[\Gamma, \Gamma^{\prime} \Rightarrow \Delta, \Delta^{\prime}\right]\right]\right.}} \mathrm{xs} / \mathrm{xtR}
$$


While reasoning from $s t$ to $s t$ is nontransitive, reasoning from $t s$ to $s t$ is transitive. To extend this observation to each standard in $\mathbb{S T}$ we shall use the following lemma:

Lemma 4.3 (Cut-elimination for $\mathbb{S T}$ ) Suppose that $x y \in \mathbb{S T}$. Then the following rule is admissible:

$$
\frac{\Gamma \Rightarrow \Delta, x: X \quad y: X, \Gamma^{\prime} \Rightarrow \Delta^{\prime}}{\Gamma, \Gamma^{\prime} \Rightarrow \Delta, \Delta^{\prime}}
$$

Proof Base case (=ST) (Simplified by omitting contexts):

$$
\Rightarrow s: A \quad \frac{t: A \Rightarrow}{s: A \Rightarrow} \mathrm{t} / \mathrm{sL}
$$

Inductive step (Simplified by omitting contexts and some branching): Assume that it holds for $x y$. Then it also holds for $y x x y$ by applying inversion and then the inductive hypothesis:

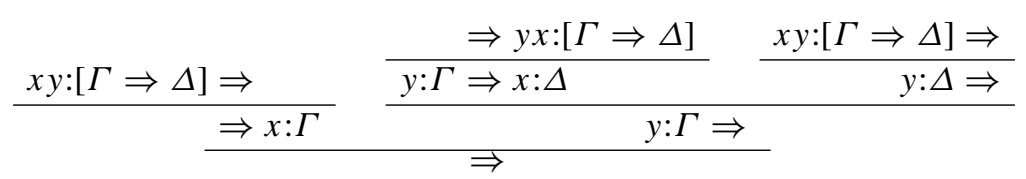

Proposition 4.4 (Every stage in $\mathbb{S T}$ is a reflexive logic) If $x y \in \mathbb{S T}$, then $\Rightarrow$ $x y:[X \Rightarrow X]$ is derivable

Proof

$$
\frac{x: X \Rightarrow x: X \quad y: X \Rightarrow y: X}{\frac{x: X \Rightarrow y: X}{\Rightarrow x y:[X \Rightarrow X]}[\Rightarrow] \mathrm{R}}
$$

Proposition 4.5 (Every stage in $\mathbb{S T}$ concerns a transitive logic) If $y x x y \in \mathbb{S T}$, then $\Rightarrow y x x y:[[\Gamma \Rightarrow \Delta, X],[X, \Gamma \Rightarrow \Delta] \Rightarrow[\Gamma \Rightarrow \Delta]]$ is derivable

Proof

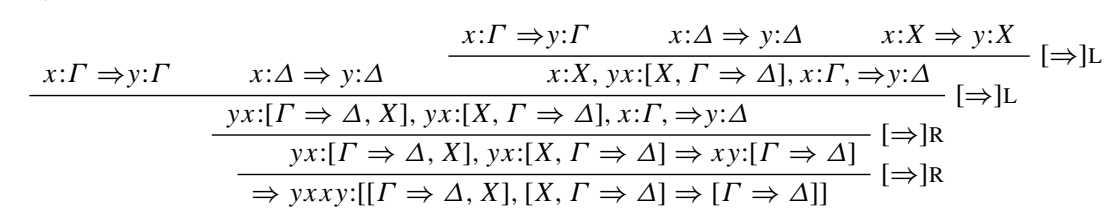

Again, while these observations are already made by [1], our proofs thereof are obtained using proof analysis. In particular, the key ingredients are our cutelimination theorem in 3.8 and the label transformation lemmas. Our proofs are thus 
arguably more elegant and easier to read than those presented involving semantic reasoning by [1].

In addition to establishing the fact that each stage is a reflexive "metainferential" logic which concerns a transitive "metainferential" logic, we can also establish that each stage is inconsistent, again using proof analysis.

Lemma 4.6 (Inconsistency is inheritable in $\mathbb{S T}$ ) For every $x y \in \mathbb{S T}$, if $x y$ is inconsistent then yxxy is inconsistent.

Proof The following pieces of reasoning are admissible:

$$
\begin{array}{cl}
\frac{\Rightarrow x y:[X \Rightarrow]}{x: X \Rightarrow} \text { Inv. of }[\Rightarrow] \mathrm{R} & \Rightarrow x y:[\Rightarrow X] \\
\frac{y x:[\Rightarrow X] \Rightarrow[\mathrm{L}}{\Rightarrow y x x y:[[\Rightarrow X] \Rightarrow]}[\Rightarrow] \mathrm{R} & \Rightarrow y x x y:[\Rightarrow[\Rightarrow X]]
\end{array}[] \mathrm{R}
$$

With $s t$ being inconsistent, the following proposition follows:

Proposition 4.7 For every $x y \in \mathbb{S T}$, $x y$ is inconsistent.

Finally, we obtain thus the following:

Proposition 4.8 (Non-classicality of every stage) For every yxxy $\in \mathbb{S T}$ the following sequents are derivable for some expression $X$ :

$$
\begin{aligned}
& \Rightarrow y x x y:[\Rightarrow[\Rightarrow X]] \quad \Rightarrow y x x y:[\Rightarrow[X \Rightarrow]] \\
& \Rightarrow y x x y:[[\Gamma \Rightarrow \Delta, X],[X, \Gamma \Rightarrow \Delta] \Rightarrow[\Gamma \Rightarrow \Delta]]
\end{aligned}
$$

The conclusion should thus not be that each level is classical to a greater extent than the previous level as we transcend up in the hierarchy as if the next level takes us closer to classical logic (even if we never reach classical logic), but rather that each stage is non-classical to the same extent. This should not be too surprising considering how each stage in the hierarchy is a $s t$-ish logic for the previous stage obtained by what amounts to a strict-tolerant standard for that stage.

\section{A "fully classical" Metainferential Logic?}

Following the observation that no stage in the $\mathbb{S T}$-hierarchy is classical, [2] presents a way to "recovers every classically valid metainference of every level". This consists in defining a collection of metainferences $\mathbb{S T} \omega$ of any level in such a way that we can understand it "as the union of" each $x \in \mathbb{S T}$. In this way then, we are supposed to obtain a "fully classical" (metainferential) logic. In the concluding remarks in [2], it is observed that 
there still is plenty work to do in relation to these logics and truth theories. For example, it seems not easy to imagine a proof theory for them [2].

As it turns out, it is straightforward to extend the HST calculus to a sequent calculus for $\mathbb{S T} \omega$. Let us thus proceed with the definitions. In [2], a definition of $\mathbb{S T} \omega$ is provided which is equivalent to the following:

Definition 5.1 Suppose that $X$ is a metainference of level $j>0$. Then $X \in \mathbb{S T} \omega$ if and only if for every $\mathcal{V}, \mathcal{V} \Vdash_{\mathrm{ST}_{j}} X$.

This definition can fruitfully be split into two stages as follows:

\section{Definition 5.2}

(a) Suppose that $X$ is a metainference of level $j>0$ and $\mathcal{V}$ is a strong Kleene valuation. Then $\mathcal{V} \Vdash_{\omega} X$ if and only if $\mathcal{V} \Vdash_{\mathrm{ST}_{j}} X$.

(b) $\quad X \in \mathbb{S T} \omega$ if and only if for every strong Kleene valuation $\mathcal{V}, \mathcal{V} \Vdash_{\omega} X$.

We proceed now to define the extended sequent calculus. To that purpose we first extend the definition of sequent expressions.

Definition 5.3 (Extended typed sequent expression) If $x: X$ is a sequent expression of type $(\alpha, x)$ where $\alpha>0$, then $x: X$ and $\omega: X$ are extended sequent expressions.

It follows that $\omega: A$ where $A$ is a formula is not an extended sequent expression whereas $\omega:[\Gamma \Rightarrow \Delta]$ where $[\Gamma \Rightarrow \Delta]$ is a metainference of any level $>0$ is an extended sequent expression.

Definition 5.4 (Extended HST calculus) Let EHST be the sequent calculus obtained by expanding the HST calculus with the following rules where $x \in \mathbb{S T}, x \neq s t$ and $X$ is a metainference of level $x$ :

$$
\frac{x: X, \Gamma \Rightarrow \Delta}{\omega: X, \Gamma \Rightarrow \Delta} \omega \mathrm{L} \quad \frac{\Gamma \Rightarrow \Delta, x: X}{\Gamma \Rightarrow \Delta, \omega: X} \omega \mathrm{R}
$$

The adequacy of the rules for $\omega$ is immediate by considering clause (a) in definition 5.2. The various lemmas and theorems for HST transfers to EHST by appropriately extending the various definitions.

Lemma 5.5 (Weakening and contraction) The following rules are HP-admissible in EHST:

$$
\frac{\Gamma \Rightarrow \Delta}{\Gamma^{\prime}, \Gamma \Rightarrow \Delta, \Delta^{\prime}} \quad \frac{x: X, x: X, \Gamma \Rightarrow \Delta}{x: X, \Gamma \Rightarrow \Delta} \quad \frac{\Gamma \Rightarrow \Delta, x: X, x: X}{\Gamma \Rightarrow \Delta, x: X}
$$

Lemma 5.6 (Inversion) The inversion of each primitive EHST-rule is HP-admissible in EHST. 
Theorem 5.7 (Cut) The following rule is admissible in EHST:

$$
\frac{\Gamma \Rightarrow \Delta, x: X \quad x: X, \Gamma^{\prime} \Rightarrow \Delta^{\prime}}{\Gamma, \Gamma^{\prime} \Rightarrow \Delta, \Delta^{\prime}}
$$

As above in the HST-calculus, cut-elimination does not imply that a metainferential logic defined with the calculus is transitive. A reasonable question to ask now is thus whether $\omega$ really is a "fully classical" metainferential logic?

Proposition 5.8 Reasoning in $\omega$ about metainferential levels in $\mathbb{S T}$ is not transitive.

Proof By proposition 4.6 it follows that for any metainferential level, there is an $X$ such that:

$$
\Rightarrow \omega:[\Rightarrow X] \quad \Rightarrow \omega:[X \Rightarrow]
$$

By cut-elimination, it follows that the sequent

$$
\omega:[\Gamma \Rightarrow \Delta, X], \omega:[X, \Gamma \Rightarrow \Delta] \Rightarrow \omega:[\Gamma \Rightarrow \Delta]
$$

is not derivable.

It follows that $\omega$ is not "more" classical than anything in $\mathbb{S T}$. In fact, we can show that $\omega$ is $s t$ in disguise.

Definition 5.9 (Notation) Let $X \longleftrightarrow Y$ mean that the following rules are admissible:

$$
\frac{X, \Gamma \Rightarrow \Delta}{Y, \Gamma \Rightarrow \Delta} \quad \frac{\Gamma \Rightarrow \Delta, X}{\Gamma \Rightarrow \Delta, Y}
$$

With the rules for introducing a metainference having the same shape as the rules for introducing a formula of the form $\left(A_{0} \wedge \ldots \wedge A_{n}\right) \supset\left(B_{0} \vee \ldots \vee B_{m}\right)$ where $\wedge$ and $\supset$ are defined as $\neg(\neg A \vee \neg B)$ and $\neg A \vee B$ respectively, it is relatively evident that we can engage in a process of flatting metainferences. For example, it follows by corollary 3.17 that the following claims hold where $\Gamma \supset \Delta$ abbreviates $\left(A_{0} \wedge \ldots \wedge\right.$ $\left.A_{n}\right) \supset\left(B_{0} \vee \ldots \vee B_{m}\right)$ where $A_{i} \in \Gamma$ and $B_{i} \in \Delta$ :

$$
\begin{aligned}
& s t:[\Gamma \Rightarrow \Delta] \longleftrightarrow t: \Gamma \supset \Delta \\
& t s:[\Gamma \Rightarrow \Delta] \longleftrightarrow s: \Gamma \supset \Delta
\end{aligned}
$$

With that established we proceed to observe the following:

$$
\begin{aligned}
& \operatorname{tsst}:\left[\left[\Gamma_{1} \Rightarrow \Delta_{1}\right], \ldots,\left[\Gamma_{n} \Rightarrow \Delta_{n}\right]\right.\left.\Rightarrow\left[\Gamma_{1}^{\prime} \Rightarrow \Delta_{1}^{\prime}\right], \ldots,\left[\Gamma_{m}^{\prime} \Rightarrow \Delta_{m}^{\prime}\right]\right] \\
& \longleftrightarrow \\
& s t:\left[\Gamma_{1} \supset \Delta_{1}, \ldots, \Gamma_{n} \supset \Delta_{n} \Rightarrow \Gamma_{1}^{\prime} \supset \Delta_{1}^{\prime}, \ldots, \Gamma_{m}^{\prime} \supset \Delta_{m}^{\prime}\right]
\end{aligned}
$$

We shall here illustrate how to establish the left rule. First, we note that for each $\left[\Gamma_{i} \Rightarrow \Delta_{i}\right]$ :

$$
\begin{aligned}
t s s t:\left[\left[\Gamma_{1} \Rightarrow \Delta_{1}\right], \ldots,\left[\Gamma_{n} \Rightarrow \Delta_{n}\right] \Rightarrow\left[\Gamma_{1}^{\prime} \Rightarrow \Delta_{1}^{\prime}\right], \ldots,\left[\Gamma_{m}^{\prime} \Rightarrow \Delta_{m}^{\prime}\right]\right] & \Rightarrow \\
\hline & \Rightarrow t s:\left[\Gamma_{i} \Rightarrow \Delta_{i}\right] \\
& \Rightarrow s: \Gamma_{i} \supset \Delta_{i}
\end{aligned}
$$


Correspondingly, we obtain that for each $\left[\Gamma_{i}^{\prime} \Rightarrow \Delta_{i}^{\prime}\right]$ :

$$
\begin{array}{r}
t s s t:\left[\left[\Gamma_{1} \Rightarrow \Delta_{1}\right], \ldots,\left[\Gamma_{n} \Rightarrow \Delta_{n}\right] \Rightarrow\left[\Gamma_{1}^{\prime} \Rightarrow \Delta_{1}^{\prime}\right], \ldots,\left[\Gamma_{m}^{\prime} \Rightarrow \Delta_{m}^{\prime}\right]\right] \Rightarrow \\
\frac{s t:\left[\Gamma_{i}^{\prime} \Rightarrow \Delta_{i}^{\prime}\right] \Rightarrow}{t: \Gamma_{i}^{\prime} \supset \Delta_{i}^{\prime} \Rightarrow}
\end{array}
$$

The desired conclusion is now obtained by the introduction an $s t$-metainference.

Let us thus provide some definitions and a more general result:

Definition 5.10 (Hierarchical reduction) If $[\Gamma \Rightarrow \Delta]$ is a metainference of level $n+1$, then $\left[\Gamma_{\supset} \Rightarrow \Delta_{\supset}\right]$ is the metainference of level $n$ obtained by replacing each metainference $\left[\Gamma^{\prime} \Rightarrow \Delta^{\prime}\right]$ of level 0 in $[\Gamma \Rightarrow \Delta]$ with the formula $\Gamma^{\prime} \supset \Delta^{\prime}$.

The above transformation of a $t s s t$-metainference to a $s t$-inference illustrates this definition in action.

Proposition 5.11 For every yxxy $\in \mathbb{S T}, y x x y:[\Gamma \Rightarrow \Delta] \longleftrightarrow x y:\left[\Gamma_{\supset} \Rightarrow \Delta_{\supset}\right]$

Proof By induction on the levels.

Definition 5.12 If $[\Gamma \Rightarrow \Delta]$ is a metainference of level $n$ then $\left[\Gamma_{\supset}^{0} \Rightarrow \Delta_{\supset}^{0}\right]$ is the result of performing an hierarchical reduction $n$ times on $[\Gamma \Rightarrow \Delta]$.

A metainference of the form $\left[\Gamma_{\supset}^{0} \Rightarrow \Delta_{\supset}^{0}\right]$ is thus simply an inference.

Theorem 5.13 ( $\omega$ is st in disguise)

$$
\omega:[\Gamma \Rightarrow \Delta] \longleftrightarrow s t:\left[\Gamma_{\supset}^{0} \Rightarrow \Delta_{\supset}^{0}\right]
$$

Proof Iterated applications of proposition 5.11.

To make sense of this theorem, it is useful to observe that $\omega$ does not, despite its label, represent a limit. Instead, it is simply a collection of metainferences of various levels. Every metainference of $\omega$ will be of a particular finite level, and can thus be reduced according to proposition 5.11. Moreover, and with that in mind, this result shouldn't actually be particularly surprising considering how the $\mathbb{S T}$-hierarchy and $\omega$ is defined and how for example $t s s t$ is the metainferential analogue of $s t$ since $t s$ is a stricter standard than $s t$ in the same way as $s$ is a stricter standard than $t$.

\section{Imitating st as Metatheory}

Since we have used the proof-theoretic tool developed in this paper to illustrate problems with the interpretation of the $\mathbb{S T}$-hierarchy proposed by [1] and [2], it seems appropriate to use the concluding remarks of this paper to engage in some speculation 
about whether we can utilise the results obtained with the tool to provide an alternative interpretation of the $\mathbb{S T}$-hierarchy, and whether this could be used to make sense of st.

The flatting of the $\mathbb{S T}$-hierarchy into st suggests that $\mathbb{S T}$-hierarchy is merely a metainferential twist on the $s t$-phenomenon, as if the $\mathbb{S T}$-hierarchy doesn't tell us anything that we couldn't already express within $s t$, and one could thus argue that the hierarchy is somehow superfluous.

On the other hand, precisely because the $\mathbb{S T}$-hierarchy is representable within $s t$, it is perhaps not too incredulous to suggest that the $\mathbb{S T}$-hierarchy can actually tell us something about how it would be to reason within $s t$ about $s t$, that is, how it would be to use $s t$ as metatheory for $s t$, and thus what $s t$ looks like from the perspective of st. Contrary to the received wisdom and thus awkwardly enough, this amounts to looking at the material conditional of $s t$ to learn more about $s t$. While some initial scepticism is certainly warranted, we have shown that we can represent the $\mathbb{S T}$-hierarchy with the material conditional in $s t$, so if the $\mathbb{S T}$-hierarchy tells us something about $s t$ as a metatheory for $s t$, then surely valid inferences about the material conditional in $s t$ would tell us what $s t$ as metatheory would tell us about $s t$.

With that in mind we can reason as follows. Under the assumption that the valid inferences about the material conditional in $s t$ represent the metainferences that hold of $s t$ within $s t$, st is transitive according to $s t$. In other words, according to $s t$ it is the case that the inferences from anything to the liar and from the liar to anything together imply that anything follows from anything. This observation corresponds to that made by [14] with regard to a validity predicate defined in $s t$ along the lines of the material conditional. From the perspective of a classical metatheory according to which $s t$ is non-transitive, that claim is false, and [14] considered their observation as presenting a problem for such an approach to defining a validity predicate in $s t$. However, we are no longer supposed to think of $s t$ from the perspective of classical logic. Instead, we are considering $s t$ from the perspective of $s t$, and what if $s t$ really is transitive when $s t$ is the metatheory for $s t$ ? After all, $s t$ is non-transitive from within a classical metatheory because assuming otherwise leads to inconsistency. With $s t$ as metatheory, however, the inconsistency is not an issue, and it follows that $s t$ can be transitive according to $s t$.

Continuing down the rabbit hole then, we also note that $s t$ tells us about $s t$ that anything implies the liar and that the liar implies anything. Now, does it follow, since those facts imply that anything implies anything, that anything implies anything? On the one hand, we can find formulas $A$ and $B$ such that $\neg(A \supset B)$ follows from no premises, a fact which is reasonably interpreted as that it is not the case that anything implies anything. On the other hand, we have cases such as $\neg(\lambda \supset \lambda)$ and $(\lambda \supset \lambda)$ which are both valid according to $s t$. Taking each statement to represent an inference as suggested above, there are thus inferences that are both valid and not valid in $s t$ according to $s t$. From the perspective of $s t$ then, it seems reasonable to think of transitivity of entailment in the same way; that it is (metainferentially) valid, but there are counterexamples. While a classically minded referee would certainly protest at this point since being valid and having a counterexample are supposed to be 'at least contraries', such a protest would just illustrate the uphill battle faced by an advocate of paraconsistent metatheory as generalised to include metainferences. Indeed, could 
the moral be that you're free to apply transitivity when reasoning within $s t$ about $s t$ as long as you're willing to accept that the conclusion you draw is a dialetheia?

Acknowledgements I would like to thank the audience at the SADAF Workshop on Metainferences and Substructural Logics in 2019 organised by the Buenos Aires Logic Group for the discussion of my presentation of the material for this paper. In addition, I am also grateful to the referees for their comments.

Funding Open access funding provided by University of Bergen (incl Haukeland University Hospital).

Open Access This article is licensed under a Creative Commons Attribution 4.0 International License, which permits use, sharing, adaptation, distribution and reproduction in any medium or format, as long as you give appropriate credit to the original author(s) and the source, provide a link to the Creative Commons licence, and indicate if changes were made. The images or other third party material in this article are included in the article's Creative Commons licence, unless indicated otherwise in a credit line to the material. If material is not included in the article's Creative Commons licence and your intended use is not permitted by statutory regulation or exceeds the permitted use, you will need to obtain permission directly from the copyright holder. To view a copy of this licence, visit http://creativecommons.org/licenses/by/4.0/.

\section{References}

1. Barrio, E.A., Pailos, F., \& Szmuc, D (2020). A hierarchy of classical and paraconsistent logics. Journal of Philosophical Logic, 49(1), 93-120. https://doi.org/10.1007/s10992-019-09513-z.

2. Pailos, F.M. (2020). A fully classical truth theory characterized by substructural means. Review of Symbolic Logic, 13(2), 249-268. https://doi.org/10.1017/s1755020318000485.

3. Ripley, D. (2013). Paradoxes and failures of cut. Australasian Journal of Philosophy, 91(1), 139-164. https://doi.org/10.1080/00048402.2011.630010.

4. Cobreros, P., Égré, P., Ripley, D., \& van Rooij, R (2013). Reaching transparent truth. Mind, 122(488), 841-866. https://doi.org/10.1093/mind/fzt110.

5. Cobreros, P., Egré, P., Ripley, D., \& van Rooij, R (2012). Tolerant, classical, strict. Journal of Philosophical Logic, 41(2), 347-385. https://doi.org/10.1007/s10992-010-9165-z.

6. Halbach, V., \& Horsten, L. (2006). Axiomatizing kripke's theory of truth. Journal of Symbolic Logic, 71(2), 677-712. https://doi.org/10.2178/js1/1146620166.

7. Scambler, C. (2020). Classical logic and the strict tolerant hierarchy. Journal of Philosophical Logic, 49(2), 351-370. https://doi.org/10.1007/s10992-019-09520-0.

8. Negri, S., \& von Plato, J. (2001). Structural proof theory. Cambridge: Cambridge University Press.

9. Negri, S. (2005). Proof analysis in modal logic. Journal of Philosophical Logic, 34, 507-544. https://doi.org/10.1007/s10992-005-2267-3.

10. Ripley, D. (2012). Conservatively extending classical logic with transparent truth. Review of Symbolic Logic, 5(2), 354-378. https://doi.org/10.1017/s1755020312000056.

11. Barrio, E., Rosenblatt, L., \& Tajer, D (2015). The logics of strict-tolerant logic. Journal of Philosophical Logic, 44(5), 551-571. https://doi.org/10.1007/s10992-014-9342-6.

12. Dicher, B., \& Paoli, F. (2019). St, lp and tolerant metainferences. In C. Bakent, \& T. Ferguson (Eds.) Graham Priest on dialetheism and paraconsistency. Springer.

13. Cobreros, P., Rosa, E.L., \& Tranchini, L (2021). (i can't get no) antisatisfaction. Synthese, pp 1-15, https://doi.org/10.1007/s11229-020-02570-X.

14. Barrio, E., Rosenblatt, L., \& Tajer, D (2021). Capturing naive validity in the cut-free approach. Synthese, pp 1-17, https://doi.org/10.1007/s11229-016-1199-5.

Publisher's Note Springer Nature remains neutral with regard to jurisdictional claims in published maps and institutional affiliations. 